\title{
Tetramelic monodactyly
}

INSERM

\section{Source}

INSERM. (1999). Orphanet: an online rare disease and orphan drug data base. Tetramelic monodactyly. ORPHA:2564

Tetramelic monodactyly is a rare, genetic, congenital limb malformation disorder characterized by the presence of a single digit on all four extremities. Malformation is typically isolated however, aplastic and hypoplastic defects in the remaining skeletal parts of hands and feet have been reported. There have been no further descriptions in the literature since 1992. 